\title{
Avaliação física de feijão carioca irradiado
}

\author{
Physical evaluation of irradiated carioca beans
}

\section{José Maurício ARMELIN ${ }^{1}$, Solange Guidolin CANNIATTI-BRAZACA ${ }^{1 *}$, Sônia Maria de Stefano PIEDADE², Flávia Maria Vasques Farinazzi MACHADO ${ }^{1}$, Marta Helena Fillet $\mathrm{SPOTO}^{1}$}

\section{Resumo}

A radiação gama é uma forma de evitar as perdas de safras de feijões e, manter a qualidade dos grãos para que o consumidor não rejeite o produto. Os objetivos desta pesquisa foram avaliar as alterações em feijão (Phaseolus vulgaris L.) variedade Carioca Tybatã submetidos a diferentes dosagens de radiação gama, com doses de 0, 1, 2, 6 e 10 kGy, verificando alterações na cor, tempo de hidratação e de cocção. A cor foi avaliada por provadores treinados em cabine de cor utilizando a luz do dia, empregando teste de ordenação. O tempo de hidratação foi determinado com 10 g de grãos para $50 \mathrm{~mL}$ de água destilada, em intervalos de medidas de 30 em 30 minutos, nas primeiras duas horas, e de 1 em 1 hora, até que a absorção se estabilizasse. O tempo de cocção foi medido utilizando equipamento para cocção. Foi utilizado Teste de Tukey (5\%) para análise dos dados obtidos com emprego do programa SAS. Não houve diferenças significativas quanto à cor dos feijões submetidos aos tratamentos com irradiação. Para o tempo de hidratação, os tratamentos não diferiram significativamente entre si ( $\mathrm{p} \leq 0,05$ ), embora, numericamente, todas as doses de irradiação tenham absorvido mais água que os grãos da amostra controle não-irradiada, com exceção da amostra que recebeu tratamento com $6 \mathrm{kGy}$. Os grãos não irradiados apresentaram significativamente maior tempo de cocção, mas não houve diferenças significativas ( $\mathrm{p} \leq 0,05)$ entre os tratamentos com irradiação 1, 2, 6 e $10 \mathrm{kGy}$.

Palavras-chave: cor; hidratação; cocção; irradiação; Phaseolus vulgaris; feijão.

\begin{abstract}
Gamma radiation is a promising treatment for the prevention of bean crop losses, preserving the quality of the product and thus avoiding its rejection by the consumer. This research evaluated the sensorial alterations in beans (Phaseolus vulgaris L.), var. Carioca Tybatã, subjected to different doses of radiation (0, 1, 2, 6 and $10 \mathrm{kGy}$ ), based on changes in color, hydration (soaking) and cooking times. The color was evaluated by trained testers in a color booth illuminated by natural daylight, using a ranking test. The hydration time was determined by soaking 10 $\mathrm{g}$ of beans in $50 \mathrm{~mL}$ of distilled water, checking their hydration at 30 minutes intervals for the first two hours and at 1 hour intervals until absorption was stabilized. The cooking time was measured using cooking equipment. Tukey's test (5\% level of significance) was applied in a statistical analysis of the data obtained with SAS software and the ranking data. The irradiated beans showed no major differences in color compared with the control (nonirradiated beans). The hydration time did not differ significantly ( $\mathrm{p} \leq 0.05$ ) among treatments, although the irradiated beans absorbed more water than the control. The nonirradiated beans took considerably longer to cook than the irradiated ones, but no statistical differences $(\mathrm{p} \leq 0.05)$ were found among the beans irradiated with $1,2,6$ and $10 \mathrm{kGy}$, nor did they show statistically significant differences in color.
\end{abstract}

Keywords: color; hydration; cooking; irradiation; Phaseolus vulgaris; beans.

\section{Introdução}

O feijão comum (Phaseolus vulgaris L.) variedade carioca é um importante alimento, devido ao rico teor de proteínas, vitaminas e minerais ( $\mathrm{Ca}, \mathrm{Fe}, \mathrm{Cu}, \mathrm{Zn}, \mathrm{P}, \mathrm{K} \mathrm{e} \mathrm{Mg})^{2,21}$.

As safras anualmente sofrem perdas significativas quando os feijões são submetidos à estocagem em condições ambientais de altas temperaturas, alta umidade relativa do meio e tempo de armazenamento prolongado, tornando-os susceptíveis aos fenômenos de endurecimento, infestação de insetos, mudanças de textura, cor e sabor, degradação de fitatos e metionina, e contribuindo para o aumento do tempo de cocção ${ }^{13}$.

Os feijões da variedade Carioca Tybatã são caracterizados por possuir alto potencial produtivo, apresentando plantas com

\footnotetext{
Recebido para publicação em 14/6/2006

Aceito para publicação em 18/7/2007 (001757)

Departamento de Agroindústria, Alimentos e Nutrição,

Escola Superior de Agricultura "Luiz de Queiroz" - ESALQ

Universidade de São Paulo - USP,

Av. Pádua Dias, 11, CP 9, CEP 13418-900, Piracicaba - SP, Brasil,

E-mail: sgcbraza@esalq.usp.br

Departamento de Ciências Exatas,

Escola Superior de Agricultura "Luiz de Queiroz" - ESALQ,

Universidade de São Paulo - USP

Av. Pádua Dias, 11, CP 9, CEP 13418-900, Piracicaba-SP, Brasil

*A quem a correspondência deve ser enviada
}

porte semi-ereto, resistentes ao vírus mosaico dourado do feijão (bean golden mosaic geminivirus) e a outros patógenos.

A irradiação gama surge como uma forma promissora para evitar essas perdas, pois garante a eliminação de microorganismos $^{11}$, promove diminuição da dureza das sementes ${ }^{9}$, diminuição do tempo de cocção, aumenta a quantidade de riboflavina, não altera a concentração de vitamina $\mathrm{B}_{6}{ }^{24} \mathrm{e}$ não provoca alteração da utilização das proteínas, bem como sua digestibilidade e seu valor biológico ${ }^{10}$. No entanto, as propriedades físicas do amido, são afetadas quando os feijões são submetidos a determinadas doses de radiação ${ }^{18,19}$. Há também alterações nutricionais e sensoriais em alimentos irradiados, porém em dimensão não superior às alterações encontradas em outros métodos de cocção.

Este processo, tendo o propósito de preservar e desinfestar grãos, pode ser utilizado para estender a vida útil e reduzir as perdas das safras durante a armazenagem do produto. Os custos estimados dos benefícios da irradiação comercial como tratamento mostram ser competitivos com outros métodos de fumigação e outros tratamentos físicos e térmicos ${ }^{17,23}$.

As doses até $10 \mathrm{kGy}$, conforme a Organização da Agricultura e Alimentos (FAO)/Organização Mundial da Saúde ${ }^{12}$, 
asseguram a inocuidade dos alimentos sob ponto de vista microbiológico, nutricional e toxicológico.

Entre as características culinárias desejáveis pelos consumidores estão a rápida hidratação, baixo tempo de cocção, produção de um caldo espesso, bom sabor e textura, grãos moderadamente rachados, casca delgada e boa estabilidade de cor. A integridade dos grãos depois de cozidos é importante para a aplicação industrial. Para a qualidade comercial dos grãos, a coloração e o tamanho são os atributos primeiramente avaliados pelos consumidores, apresentando papel decisivo na aceitação do produto ${ }^{5}$.

As qualidades dos grãos devem ser mantidas para que o consumidor não rejeite o produto, sendo, portanto, necessário avaliar as alterações sensoriais de feijões (Phaseolus vulgaris L.), variedade Carioca Tybatã, submetidos a diferentes dosagens de radiação gama, doses de 0, 1, 2, 6 e 10 kGy, quanto a mudanças na coloração, bem como tempo de hidratação e de cocção.

\section{Material e métodos}

\subsection{Matéria-prima}

Para as análises, foram utilizados grãos de feijões provenientes da cultivar-IAC-Carioca Tybatã.

Os feijões foram irradiados com raios gama provenientes do Cobalto-60 em doses de 0 (Controle), 1, 2, 6 e 10 kGy, aos 10 dias pós-colheita, permanecendo armazenados em sacos plásticos em geladeira até a conclusão dos testes.

A irradiação foi conduzida em irradiador tipo Gammacell da Nordion com atividade de 56,21 TBq no Centro de Energia Nuclear na Agricultura (CENA/USP), na cidade de Piracicaba/ SP. O processo foi conduzido em câmara especial de processamento por tempo determinado. A taxa de dose utilizada foi de $1,6 \mathrm{kGy} / \mathrm{h}$.

\subsection{Tempo de hidratação}

O tempo de hidratação dos grãos foi determinado à temperatura ambiente com $10 \mathrm{~g}$ de grãos para $50 \mathrm{~mL}$ de água destilada, de 30 em 30 minutos nas 2 primeiras horas e, depois, com intervalos de 1 em 1 hora até que a absorção se estabilizasse $^{15}$.

Os dados foram avaliados segundo o teste de Tukey ( $\mathrm{p} \leq 0,05)$ com a utilização do programa SAS ${ }^{22}$.

\subsection{Tempo de cocção}

Os grãos foram embebidos em água destilada por 10 horas à temperatura ambiente. O tempo de cocção foi medido pelo uso de equipamento de cocção de Mattson, conforme o proposto por BURR et al. ${ }^{6}$. Foram retirados de cada amostra macerada 25 grãos de feijões, os quais foram coletados ao acaso, sendo, contudo, descartados os grãos com tegumento rompido ou ausente. O tempo ótimo de cocção foi definido como o tempo necessário para que 13 hastes perfurassem completamente os grãos. Estes testes foram realizados no Centro de Energia Nuclear na Agricultura da Universidade de São Paulo (CENA/USP).

Os dados foram avaliados segundo o teste de Tukey ( $\mathrm{p} \leq 0,05)$ com a utilização do programa $\mathrm{SAS}^{22}$.

\subsection{Análise de cor}

Os testes foram realizados no Laboratório de Análise Sensorial do Departamento de Agroindústria, Alimentos e Nutrição, da Escola Superior de Agricultura "Luiz de Queiroz", em cabines individuais, com utilização de luz branca, longe de ruídos e odores.

O método empregado para análise de cor foi o teste de ordenação ${ }^{14}$. Cada provador avaliou a cor das 5 amostras de feijões (controle, 1, 2, 6 e $10 \mathrm{kGy}$ ) ordenando-as de modo crescente de tonalidades de marrom, fazendo o registro em fichas, utilizando cinco níveis de cor, iniciado com a cor "marrom claro" e finalizado com a cor "marrom escuro".

As amostras de feijões, depois de cozidas com base no método de cocção empregado de panela aberta pelo tempo obtido conforme descrito no item 2.3, foram dispostas aleatoriamente em forma de Quadrado Latino ${ }^{8}$ para cada participante selecionado, em pratos de porcelana branco, codificados com números de 3 dígitos. A avaliação foi realizada no interior da cabine para análise de cor (Colorchecker modelo executive), o qual possui sistema de iluminação com emissão de luz, temperatura de cor balanceada com capacidade para simular as três fontes de luz padrão utilizadas mundialmente (Luz do dia-artificial TL-86 a 6500K; Luz fluorescente branca fria - 4000K ou TL-84 com o mínimo de teor de radiação em luz vermelha e a luz do horizonte ou incandescente). A luz que foi utilizada na condução dos testes foi a luz do dia.

Para a análise dos resultados, foi empregada a Tabela de Newell e MacFarlane ${ }^{4}$, na qual as diferenças das somas dos tratamentos foram confrontadas com a tabela e, quando a $\mathrm{DS}_{\mathrm{T}}$ (diferença da soma entre os resultados de todos os provadores para dois tratamentos) foi maior do que a diferença tabelada, a diferença entre esses tratamentos foi considerada positiva estatisticamente.

\section{Resultados e discussão}

\subsection{Tempo de hidratação}

A Tabela 1 mostra a quantidade de água absorvida em $\mathrm{mL}$ pelos grãos ao atingirem a estabilização da hidratação. Todos os tratamentos controle (0), 1, 2, 6 e 10 kGy não diferiram significativamente entre si ao nível de $(\mathrm{p} \leq 0,05)$. No entanto, numericamente as doses de irradiação 1, 2 e 10 kGy absorveram mais água que os grãos da amostra controle, sendo que a dose de irradiação 6 kGy absorveu a mesma quantidade de água da amostra controle.

Observando a Figura 1, para o controle, os feijões atingiram em menor tempo a estabilização da hidratação (4 horas de teste), absorvendo um total de $13 \mathrm{~mL}$ de água. Os grãos subme- 
Tabela 1. Quantidade total de água absorvida (mL) e tempo de estabilização (horas) pelos grãos de feijões irradiados com 0, 1, 2, 6 e 10 kGy.

\begin{tabular}{lcc}
\hline Tratamentos & $\begin{array}{c}\text { Quantidade de água absorvi- } \\
\text { da (mL) até estabilização }\end{array}$ & $\begin{array}{c}\text { Tempo para } \\
\text { estabilização (horas) }\end{array}$ \\
\hline Controle & $13,00^{1} \pm 1,7^{\mathrm{a} 2}$ & 4 \\
$1 \mathrm{kGy}$ & $14,00 \pm 1,7^{\mathrm{a}}$ & 7 \\
$2 \mathrm{kGy}$ & $13,67 \pm 1,5^{\mathrm{a}}$ & 8 \\
$6 \mathrm{kGy}$ & $13,00 \pm 0,0^{\mathrm{a}}$ & 4 \\
$10 \mathrm{kGy}$ & $16,33 \pm 1,1^{\mathrm{a}}$ & 8 \\
\hline
\end{tabular}

${ }^{1}$ Média \pm desvio padrão; $\mathrm{e}^{2}$ médias com letra(s) diferente(s) na vertical diferem significativamente ao nível de $(\mathrm{p} \leq 0,05)$.

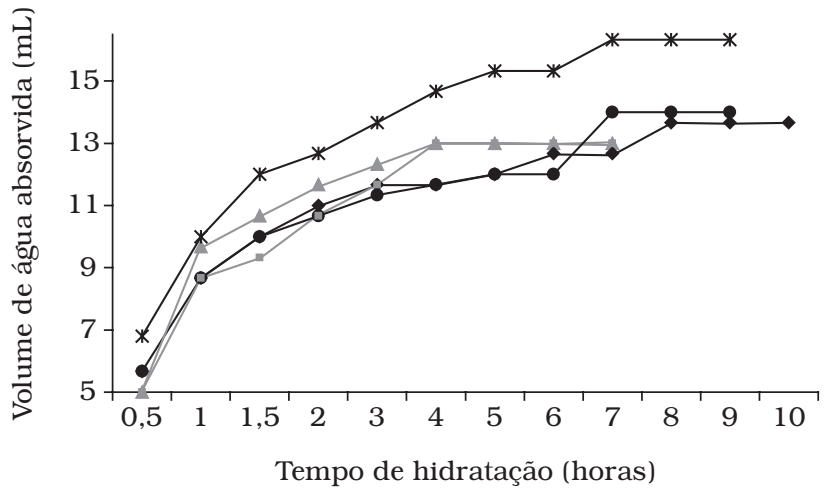

$$
\multimap 0 \mathrm{kGy} \multimap 1 \mathrm{kGy} \multimap 2 \mathrm{kGy} \rightleftarrows 6 \mathrm{kGy} \rightarrow-10 \mathrm{kGy}
$$

Figura 1. Efeito da irradiação sobre a capacidade de hidratação dos grãos de feijão.

tidos aos tratamentos de 2 e $10 \mathrm{kGy}$ levaram mais tempo para estabilização, quando comparados aos demais tratamentos.

Segundo CARVALHO et al. ${ }^{7}$, feijões endurecidos irradiados com 500 e 1.000 Gy, aumentaram a velocidade de hidratação em relação aos não irradiados. Do mesmo modo, segundo CUNHA et al. ${ }^{9}$, feijões irradiados com $2 \mathrm{kGy}$, submetidos a diferentes condições de armazenamento, aumentaram também a taxa de hidratação.

\subsection{Tempo de cocção}

A Tabela 2 apresenta o tempo de cocção em minutos e a Figura 2 expressa os tempos em que as diferentes hastes perfuraram os grãos ( 1 a 13) em minutos, para os grãos de feijão irradiados e não-irradiados. A irradiação diminuiu numericamente, em média, considerando-se os 4 tratamentos, $1 / 3$ do tempo de cozimento em relação aos não-irradiados. Não houve diferenças significativas ( $p \leq 0,05)$ entre os tratamentos com 1, 2, 6 e $10 \mathrm{kGy}$. O controle diferiu significativamente ( $\mathrm{p} \leq 0,05$ ) dos irradiados, apresentando maior tempo de cocção. Resultados semelhantes foram obtidos por CARVALHO et al. ${ }^{7}$, empregando irradiação com 500 e 1000 Gy.

CUNHA et al. ${ }^{9}$ também obtiveram redução no tempo de cocção de grãos de feijão irradiados com 2 kGy, até no sexto mês de estocagem, sob refrigeração $\left(4-5^{\circ} \mathrm{C}\right)$ ou a $30^{\circ} \mathrm{C}, 75 \%$ UR.
Tabela 2. Tempo de cocção dos feijões irradiados com $0,1,2,6$ e 10 kGy.

\begin{tabular}{cc}
\hline Tratamentos & Tempo de cocção (minutos) \\
\hline Controle & $40,34^{1} \pm 6,5^{\mathrm{a} 2}$ \\
$1 \mathrm{kGy}$ & $24,17 \pm 1,3^{\mathrm{b}}$ \\
$2 \mathrm{kGy}$ & $22,62 \pm 5,1^{\mathrm{b}}$ \\
$6 \mathrm{kGy}$ & $15,23 \pm 1,1^{\mathrm{b}}$ \\
$10 \mathrm{kGy}$ & $14,00 \pm 3,1^{\mathrm{b}}$ \\
\hline${ }^{\mathrm{I}}$ Média \pm desvio padrão; ${ }^{2}$ médias com letra(s) diferente(s) na vertical diferem significativamente
\end{tabular}
ao nível de $(\mathrm{p} \leq 0,05)$

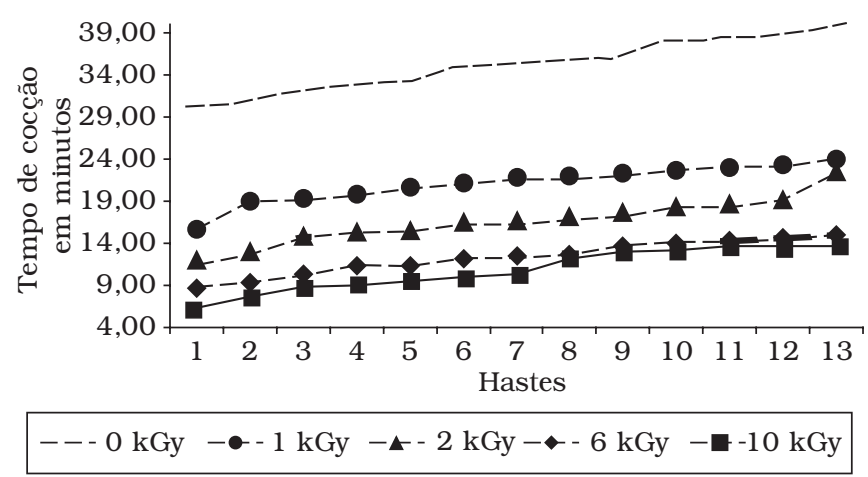

Figura 2. Efeito da irradiação sobre a cocção dos grãos de feijão.

A refrigeração cooperou ainda mais para reduzir os tempos de cocção dos grãos irradiados em relação ao controle. Da mesma forma, foi verificado o efeito da irradiação no tempo de cocção de feijões por VILLAVICENCIO et al. ${ }^{24}$, aplicando doses de 0 , $0,5,1,2,5,5$ e $10 \mathrm{kGy}$, e armazenando os grãos à temperatura ambiente durante 6 meses antes da cocção. Os autores encontraram declínios nos tempos de cozimento conforme aumento nas doses de irradiação, acompanhados de perdas na qualidade sensorial.

\subsection{Análise de cor}

Pela Tabela de Newell e MacFarlane ${ }^{4}$, a diferença crítica entre os totais de ordenação em nível de $5 \%$, para 12 julgamentos e 5 amostras, é 22. Portanto, todas as amostras que diferirem entre si por um valor maior ou igual a 22, são significativamente diferentes $(\mathrm{p} \leq 0,05)$. A Tabela 3 mostra os resultados obtidos.

Tabela 3. Resultados da ordenação da cor dos feijões irradiados com $0,1,2,6$ e $10 \mathrm{kGy}$.

\begin{tabular}{cc}
\hline Dose $(\mathrm{kGy})$ & Soma (notas) \\
\hline Controle $(0)$ & $27^{\mathrm{a}}$ \\
Dose $1 \mathrm{kGy}$ & $39^{\mathrm{a}}$ \\
Dose $2 \mathrm{kGy}$ & $30^{\mathrm{a}}$ \\
Dose $6 \mathrm{kGy}$ & $40^{\mathrm{a}}$ \\
Dose $10 \mathrm{kGy}$ & $44^{\mathrm{a}}$ \\
\hline
\end{tabular}

Comparando-se as amostras:

- Tratamento Controle (0 kGy) - Tratamento com $1 \mathrm{kGy}=$ $27-39=12$ 
- Tratamento Controle (0 kGy) - Tratamento com 2 kGy = $27-30=3$

- Tratamento Controle (0 kGy) - Tratamento com 6 kGy = $27-40=13$

- Tratamento Controle ( 0 kGy) - Tratamento com $10 \mathrm{kGy}=$ $27-44=17$

- Tratamento com $1 \mathrm{kGy}$ - Tratamento com $2 \mathrm{kGy}=$ $39-30=9$

- Tratamento com $1 \mathrm{kGy}$ - Tratamento com $6 \mathrm{kGy}=$ $39-40=1$

- Tratamento com $1 \mathrm{kGy}$ - Tratamento com $10 \mathrm{kGy}=$ $39-44=5$

- Tratamento com $2 \mathrm{kGy}$ - Tratamento com $6 \mathrm{kGy}=$ $30-40=10$

- Tratamento com 2 kGy - Tratamento com $10 \mathrm{kGy}=$ $30-44=14$

- Tratamento com 6 kGy - Tratamento com $10 \mathrm{kGy}=$ $40-44=4$

Diante dos resultados estatísticos, pode-se observar que não houve diferença significativa quanto à cor dos feijões submetidos aos tratamentos com irradiação.

MOURA et al. ${ }^{16}$, submetendo feijão preto à radiação de cobalto-60, observaram que não ocorreu alteração de cor entre o feijão preto não irradiado e os irradiados em diferentes doses. O mesmo sendo observado por ARMELIN et al. ${ }^{3}$, que constataram que a irradiação não alterou significativamente na aparência os atributos de uniformidade da cor e viscosidade, e para o odor, não alterou o odor adocicado.

Por outro lado, RIOS et al. ${ }^{20}$ notaram escurecimento no tegumento de feijões não irradiados após 6 meses de armazenamento em temperatura ambiente. Segundo ABREU e RAMALHO $^{1}$, com o tempo de armazenamento, a cor dos feijóes escurece e, depois de certo período armazenado, os grãos apresentam problemas no cozimento.

\section{Conclusóes}

A irradiação empregada em suas diferentes doses não alterou a capacidade de hidratação dos grãos, quando comparada ao controle. Porém, os grãos de feijões irradiados obtiveram redução significativa no tempo de cocção, comparando-se ao controle.

O teste de ordenação aplicado sobre a cor dos feijões demonstrou que a irradiação não interferiu a sua coloração.

\section{Agradecimentos}

Ao Departamento de Produção Vegetal da Escola Superior de Agricultura "Luiz de Queiroz" (ESALQ/USP) e ao Instituto Agronômico de Campinas (IAC) pela doação dos grãos de feijão.

\section{Referências bibliográficas}

1. ABREU, A. F. B.; RAMALHO, M. A. P. Cultivo do Feijão Irrigado na Região Noroeste de Minas Gerais. Embrapa: Sistemas de Produção, n. 5, 2005
2. ANTUNES, P. L.; SGARBIERI, V. C. Fatores antinutricionais, toxicidade e valor nutricional do feijão comum (Phaseolus vulgaris L.). Agros, v. 15, n. 1, p. 39-62, 1980.

3. ARMELIN, J. M. et al. Quantitative descriptive analysis of common bean (Phaseolus vulgaris L.) under gamma radiation. Journal of Food Science, v. 71, n. 1, p. S8-S12, 2006.

4. ASSOCIAÇÃO BRASILEIRA DE NORMAS TÉCNICAS NBR 13170tabela de Newell e MacFarlane, 1994.

5. BRESSANI, R. Revisión sobre la calidad del grano de frijol. Archivos Latinoamericanos de Nutrición, Guatemala, v. 39, n. 3, p. 419-442, sep. 1989.

6. BURR, H. R.; KON, S.; MORRIS, H. J. Cooking rates of dry beans as influenced by moisture content and temperature and time of storage. Food Technology, v. 22, n. 3, p. 336-338, 1968.

7. CARVALHO, M. R. B. et a. Efeitos da irradiação (raios $\gamma$ ) sobre as propriedades físicas, sensoriais e nutritivas dos grãos de feijão Pesquisa Agropecuária Brasileira v. 26, n. 10, p. 1663-1.672, 1991.

8. COCHRAN, W. G.; COX, G. M. Experimental design. $2^{\text {a }}$ ed. New York: John Wiley, 1992. $611 \mathrm{p}$.

9. CUNHA, M. F.; SGARBIERI, V. C.; DAMÁSIO, M. H. Effects of pretreatment with gamma rays or microwaves on storage stability of dry beans Journal Agricultural Food Chemistry, v. 41, n. 10, p. 1710-1715, 1993.

10. DELINCÉE, H.; VILLAVINCENCIO, A. L. C. H.; MANCINI-FILHO, J. Protein quality of irradiated brasilian beans. Radiation Physics and Chemistry. v. 52, n. 1-6, p. 43-46, 1998.

11. DOGBEVI, M. K.; VACHON, C.; LACROIX, M. Effect of gamma irradiation on the microbiological quality and on the functional properties of protein in dry red kidney beans (Phaseolus vulgaris) Radiation Physics and Chemistry, v. 57, n. 3-6, p. 265-268, 2000.

12. FAO/OMS. Codex Alimentarius: requisitos generales. 2. ed. Roma, 2000. v. 1 A. 400 p.

13. MARTÍN-CABREJAS, M. A. et al. Changes in physicochemical properties of dry beans (Phaseolus vulgaris L.) during long-term storage. Journal Agricultural Food Chemistry, v. 45, n. 8, p. 3223-3227, 1997.

14. MEILGAARD, M.; CIVILLE, G. V.; CARR, B. T. Sensory evaluation techniques, 3 ed. Boca Raton, Florida: CRC Press, 1987. $387 \mathrm{p}$.

15. MORRIS, H. J.; OLSON, R. L.; BEAN, R. C. Processing quality of varieties and strains of dry beans. Food Technology, v. 4, n. 7, p. 247-251, 1950.

16. MOURA, N. C. et al. Avaliação sensorial de feijão preto submetido à radiação de cobalto-60. Ciência e Tecnologia de Alimentos, v. 25, n. 2 , p. $370-374,2005$.

17. NASCIMENTO, L. M. Efeito da radiação gama $\left({ }^{60} \mathrm{Co}\right)$ nas propriedades físico-químicas e sensoriais de feijões envelhecidos (Phaseolus vulgaris). São Paulo, 1992. 135 p. Tese (Doutorado) Faculdade de Ciências Farmacêuticas, Universidade São Paulo.

18. RAYAS-DUARTE, P. et al. Effect of gamma irradiation on chemical and physical properties of dry bean (Phaseolus vulgaris) starch. Cereal Foods World v. 32, n. 9, p. 659-660, 1987.

19. RAYAS-DUARTE, P.; RUPNOW, J. H. Gamma-irradiation affects some physical properties of dry bean (Phaseolus vulgaris) starch. Journal of Food Science, v. 58, n. 2, p. 389-394, 1993.

20. RIOS, A. O.; ABREU, C. M. P.; CORRÊA, A. B. Efeitos da época de colheita e do tempo de armazenamento no escurecimento do 
tegumento do feijão (Phaseolus vulgaris L.). Ciência Agrotécnica, v. 26 , n. 3, p. 550-558, 2002.

21. ROSTON, A. J. Feijão. Campinas: CATI, 1990. 18 p. (Boletim Técnico, 199).

22. SAS Statistical Analysis System (SAS Institute Inc., release 8.2 1999-2001).

23. VILLAVICENCIO, A. L. C. H. Avaliação dos efeitos da radiação ionizante de $60 \mathrm{Co}$ em propriedades físicas, químicas e nutricionais dos feijões Phaseolus vulgaris L. e Vigna unguiculata (L.) Walp. São Paulo, 1998. 138 p. Tese (Doutorado) - Faculdade de Ciências Farmacêuticas, Universidade de São Paulo.

24. VILLAVICENCIO, A. L. C. H. et al. Effect of gamma irradiation on the thiamine, riboflavin and vitamin $\mathrm{B}_{6}$ content in two varieties of brazilian beans. Radiation Physics and Chemistry, v. 57, n. 3-6, p. 299-303, 2000 